Technological University Dublin

DÜBLIN

ARROW@TU Dublin

Articles

School of Mathematics

1989-01-01

\title{
Maximal order abelian subgroups of symmetric groups
}

J. M. Burns

Brendan Goldsmith

Technological University Dublin, brendan.goldsmith@tudublin.ie

Follow this and additional works at: https://arrow.tudublin.ie/scschmatart

Part of the Mathematics Commons

\section{Recommended Citation}

Burns, J. M., \& Goldsmith, B. (1989). Maximal order abelian subgroups of symmetric groups. Journal of the London Mathematical Society, vol. 21, pg. 70-72. doi:10.1112/blms/21.1.70

This Article is brought to you for free and open access by the School of Mathematics at ARROW@TU Dublin. It has been accepted for inclusion in Articles by an authorized administrator of ARROW@TU Dublin. For more information, please contact arrow.admin@tudublin.ie, aisling.coyne@tudublin.ie,gerard.connolly@tudublin.ie.

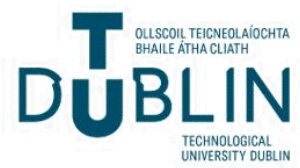




\title{
MAXIMAL ORDER ABELIAN SUBGROUPS OF SYMMETRIC GROUPS
}

\author{
J. M. BURNS AND B. GOLDSMITH
}

\section{Introduction}

Colleagues working in the area of statistical mechanics recently posed the following question: 'Are all Abelian subgroups of maximal order in the symmetric group $S_{n}$ isomorphic?' Their problem arose from considerations of reducible representations constructed from tensor products of unitary representations arising in the study of the statistical mechanics of systems of $n$ quantum spins. In particular they wanted to know what the situation was as $n \rightarrow \infty$. A complete classification of the maximal order Abelian subgroups of $S_{n}$ can be derived from recent general results of Kovacs and Praeger [2]; our objective here, however, is to give a straightforward proof of this classification using the concept of the trace of an Abelian group introduced recently by Hoffman [1]. Our notation will be standard; in particular a cyclic group of order $n$ will be denoted by $Z_{n}$ and the product of $k$ copies of such a group will be denoted by $Z_{n}^{k}$. Our principal result is:

THEOREM 1. Let $G$ be an Abelian subgroup of maximal order of the symmetric group $S_{N}$. Then

(i) $G \cong Z_{3}^{k}$ if $N=3 k$,

(ii) $G \cong Z_{2} \times Z_{3}^{k}$ if $N=3 k+2$,

(iii) either $G \cong Z_{4} \times Z_{3}^{k-1}$ or $G \cong Z_{2} \times Z_{2} \times Z_{3}^{k-1}$ if $N=3 k+1$.

\section{The trace of a finite abelian group}

As is well known, any finite Abelian group $G$ can be expressed (in multiplicative notation) as a direct product of cyclic groups of prime power order,

$$
G=Z_{m_{1}} \times Z_{m_{2}} \times \ldots \times Z_{m_{k}}
$$

and the unordered sequence $\left(m_{1}, m_{2}, \ldots, m_{k}\right)$ completely determines (up to isomorphism) $G$. Then, as observed in Hoffman [1], any symmetric function of the $m_{i}$ is an invariant of $G$. In particular the function $T(G)=\sum_{i=1}^{k} m_{i}$ is an invariant, the trace of $G$. (Note that if $G$ is trivial then we set $T(G)=0$.) We shall use the following result from [1].

Proposition 2. If an Abelian group $G$ is imbedded in the symmetric group $S_{n}$ then $T(G) \leqslant n$.

Received 5 January 1988.

1980 Mathematics Subject Classification 20B35.

Bull. London Math. Soc. 21 (1989) 70-72 
Notice that the converse is trivially true. Thus it follows that the problem of determining the maximal order Abelian subgroups of a symmetric group $S_{N}$ reduces to solving:

Maximize the product $\prod m_{i}$ of the prime powers $m_{i}$ subject to the constraint $\sum m_{i} \leqslant N$.

It is in this form that we tackle the problem.

\section{The classification}

Before presenting the combinatorial solution we note the following heuristical argument. It is clear (see Lemma 3 below) that the constraint $\leqslant N$ in (*) is rather spurious; maximality will require equality. A standard application of Lagrange multiplier techniques would indicate that all (or as many as possible) of the integers $m_{i}$ should be chosen equal (to $m$ say). Thus we want to maximize an expression of the form $m^{k}$ subject to $\mathrm{km}=N$. But this amounts to maximizing $m^{N / m}$ and, regarding this as a real function, an elementary calculation gives this function as having a maximum at $e$ and it is decreasing from $e$ onwards. Moreover since $2^{N / 2}<3^{N / 3}$ we would expect the solution of the integer-valued problem to be of the form $3^{k}$. We now give a rigorous demonstration of Theorem 1 .

LEMMA 3. The solution to (*) occurs when $\sum m_{i}=N$.

Proof. If $\sum_{i=1}^{k} m_{i}<N$ let $N-\sum_{i=1}^{k} m_{i}=t$ where $t \geqslant 1$. Then

$$
m_{1}+t=p_{1}^{r_{1}} \ldots p_{s}^{r_{s}}=p_{1}^{r_{1} u}
$$

where the $p_{i}$ are primes. Then the prime powers $m_{2}, \ldots, m_{k}, q_{1}, \ldots, q_{u}$ (where each $q_{i}=p_{1}^{r_{1}}$ ) have sum $N$ but their product exceeds $\prod_{i=1}^{k} m_{i}$ since

$$
\left(p_{1}^{r_{1}}\right)^{u} \geqslant u\left(p_{1}^{r_{1}}\right)=m_{1}+t>m_{1} .
$$

Suppose the prime powers $2^{t_{1}}, 2^{t_{2}}, \ldots, 2^{t_{l}}, \lambda_{1}, \lambda_{2}, \ldots, \lambda_{k}$ are a solution of:

$$
\text { Maximize } \prod m_{i} \text { subject to } \sum m_{i}=N \text {. }
$$

Then we have the following.

LEMMA 4. The terms $\lambda_{i}(1 \leqslant i \leqslant k)$ are all powers of 3 .

Proof. Suppose, without loss of generality, that $\lambda_{1}=p^{t}$ where $p$ is a prime $\geqslant 5$. Let $n=p^{t-1} \geqslant 1$ and note that $n \geqslant t$. Then we may write $p=3 s+r$ where $s \geqslant 1$ and $r=1,2$. If $r=2$ and we replace $\lambda_{1}$ by $q_{1}=q_{2}=\ldots=q_{n s}=3, m_{1}=m_{2}=\ldots=m_{n}=2$ then the set of prime powers $2^{t_{1}}, \ldots, 2^{t_{l}}, \lambda_{2}, \ldots, \lambda_{k}, m_{1}=\ldots=m_{n}, q_{1}=\ldots=q_{n s}$ has sum $N$ but has product which exceeds $2^{\Sigma t_{i}} \prod \lambda_{i}$ since $3^{s n} 2^{n}=\left(3^{s} 2\right)^{n}>(3 s+2)^{n} \geqslant p^{t}$ since $n \geqslant t$-contradiction. However if $r=1$, (note then that $s \geqslant 2$ ) and we replace $\lambda_{1}$ by $q_{1}=\ldots=q_{n \varepsilon}=3$ then the terms $2^{t_{1}}, \ldots, 2^{t_{t}}, \lambda_{2}, \ldots, \lambda_{k}, q_{1}, \ldots, q_{n s}$ have sum $\leqslant N$. Thus, although this set may not be an optimal choice, its product exceeds the supposed maximal product since $3^{n s}>(3 s+1)^{n}$ (because $\left.s \geqslant 2\right)$ and $(3 s+1)^{n}=p^{n} \geqslant p^{t}$ -contradiction. This contradiction to the maximality property in $(* *)$ establishes the lemma.

LEMMA 5. (i) $t_{i} \leqslant 2$ for each $i$; (ii) $\sum_{i=1}^{l} t_{i} \leqslant 2$. 
Proof. (i) Assume, without loss of generality, that $t_{1}>2$ and express $2^{t_{1}}=3 \mathrm{~s}+r$ where $r=1,2$ and $s \geqslant 2$. Then if $r=2$ replace the term $2^{t_{1}}$ by $q_{1}=\ldots=q_{s}=3$ and $m_{1}=2$. These terms have sum $2^{t}$ but their product is $3^{s} \cdot 2$ which exceeds $3 s+2$, giving a contradiction. Finally if $r=1$ replace $2^{t_{1}}$ by $q_{1}=\ldots=q_{s}=3$. The resulting system, which is not optimal since it has sum $N-1$, has a product which exceeds the supposed maximal product since $3^{s}>3 s+1$ (because $s \geqslant 2$ ), again a contradiction. Thus we have established part (i) of the Lemma.

Assume in (ii) that $\sum_{i=1}^{l} t_{i}>2$ and express this sum as $3 s+r$. We consider separately the three possible cases $s=0,1,2$. Observe firstly however that $\sum_{i=1}^{l} 2^{t_{i}}=2 \sum_{i=1}^{l} t_{i}$ if each $t_{i} \leqslant 2$.

(a) If $r=0$ replace $2^{t_{1}}, \ldots, 2^{t_{l}}$ by $q_{1}=\ldots=q_{2 s}=3$ and note that

$$
\sum_{i=1}^{l} 2^{t_{i}}=6 s=q_{1}+\ldots+q_{2 s} \text {. }
$$

However since $3^{2 s}>2^{3 s}$ this would contradict the maximality property in (**).

(b) If $r=1$ replace $2^{t_{1}}, \ldots, 2^{t_{l}}$ by $q_{1}=\ldots=q_{2 s}=3, m_{1}=2$. Again this leaves the sum unchanged but the new product would exceed the supposed maximal product since $3^{2 s} \cdot 2>2^{3 s+1}$.

(c) This case is handled exactly as in (b) only using $m_{1}=2^{2}$.

LEMMA 6. The maximum power of 3 occurring in any term $\lambda_{i}$ is 1 .

Proof. Suppose, without loss of generality, that $\lambda_{1}=3^{k}$ where $k>1$. Then replace the set $\left\{\lambda_{1}, \ldots, \lambda_{k}\right\}$ by $\left\{m_{0}, m_{1}, m_{2}, \lambda_{2}, \ldots, \lambda_{k}\right\}$ where $m_{0}=m_{1}=m_{2}=3^{k-1}$. The set $\left\{m_{0}, m_{1}, m_{2}, \lambda_{2}, \ldots, \lambda_{k}, 2^{t_{1}}, \ldots, 2^{t_{l}}\right\}$ satisfies the additive condition in (**) but $m_{0} m_{1} m_{2}=3^{3 k-3}>3^{k}$ if $k>1$. So we must conclude $k=1$.

The classification now follows immediately from an examination of the congruence of $N$ modulo 3 using the fact that $3^{k-1} \cdot 4>3^{k} \cdot 1$.

\section{References}

1. M. Hoffman, 'An invariant of finite abelian groups', Amer. Math. Monthly 94 (1987) 664-666.

2. L. G. Kovacs and C. E. PraEger, 'Finite permutation groups with large abelian quotients', Research Report 13 (1987) Australian National University Mathematics Research Report Series.

Dublin Institute of Technology

Kevin Street

Dublin 8

Ireland

\author{
Dublin Institute of Technology \\ Kevin Street \\ Dublin 8 \\ Ireland
}

and

Dublin Institute for Advanced Studies

Burlington Road

Dublin 4

Ireland 